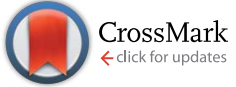

Cite this: J. Anal. At. Spectrom., 2015, 30, 296

Received 19th September 2014 Accepted 11th November 2014

DOI: $10.1039 / c 4 j a 00313 f$

www.rsc.org/jaas

\section{Direct analysis of dried blood spots by femtosecond-laser ablation-inductively coupled plasma-mass spectrometry. Feasibility of split-flow laser ablation for simultaneous trace element and isotopic analysis}

\begin{abstract}
M. Aramendia, ${ }^{a b}$ L. Rello, ${ }^{c}$ S. Bérail, ${ }^{d}$ A. Donnard, ${ }^{d}$ C. Pécheyran ${ }^{d}$ and M. Resano ${ }^{* b}$
This work describes a novel procedure based on the use of a $1030 \mathrm{~nm}$ femtosecond (fs) laser ablation (LA) device operating at a high repetition rate $(30000 \mathrm{~Hz})$ coupled to a sector field-inductively coupled plasmamass spectrometer (ICP-MS), enabling the complete ablation of dried blood spot (DBS) specimens in a reasonable time $(200 \mathrm{~s}$ for samples of $5 \mu \mathrm{L}$ ). The integration of the complete signal obtained, in combination with the use of Pt as an internal standard (which can be added to the clinical filter paper prior to the blood deposition, ensuring compatibility with unsupervised sample collection schemes), permits obtaining an analytical response that is independent of the particular characteristics of every sample. On the basis of this methodology, an analytical method was developed for the direct determination of several elements $(\mathrm{Cd}, \mathrm{Co}, \mathrm{Cu}$ and $\mathrm{Pb}$ ) in four blood reference materials as well as in three real samples, providing accurate results in all cases evaluated, at concentration levels ranging from 0.1 to hundreds of $\mu \mathrm{g} \mathrm{L}^{-1}$. Detection limits of $0.043(\mathrm{Cd}), 0.42(\mathrm{Co}), 0.54(\mathrm{Cu})$, and $0.040(\mathrm{~Pb}) \mu \mathrm{g} \mathrm{L}^{-1}$ are achieved, and precision values most often range between 3 and $9 \%$ RSD. Finally, the potential to couple the LA device simultaneously to a multicollector-ICP-MS and a sector field-ICP-MS unit by split-flow is also demonstrated, thus allowing us to obtain both elemental ( $\mathrm{Co}, \mathrm{Cu}, \mathrm{Cd}$ and $\mathrm{Pb}$ ) and isotopic (Cu isotopic composition) information from every particular DBS, and therefore maximizing the amount of information that can be drawn from a single DBS specimen. Still, the precision of the approach is limited at this point, as RSD values of approx. 1500 ppm and delta variations of almost 4\% were observed for five DBS specimens created from the same blood sample.
\end{abstract}

\section{Introduction}

In 1963, capillary blood dried on a filter paper (FP) was introduced as a clinical specimen in the U.K. to screen for phenylketonuria in newborns. ${ }^{1}$ Since that moment, large scale neonatal screening programs based on the use of dried blood spots (DBS) have been implemented throughout the world, testing for more than thirty disorders and contributing to saving and improving many lives. ${ }^{2}$

In the last few years, the importance of DBS related research has grown exponentially with more than 1200 articles published

${ }^{a}$ Centro Universitario de la Defensa-Academia General Militar de Zaragoza, Carretera de Huesca s/n, 50090, Zaragoza, Spain

${ }^{b}$ Department of Analytical Chemistry, Aragón Institute of Engineering Research (I3A), University of Zaragoza, Pedro Cerbuna 12, 50009, Zaragoza, Spain. E-mail: mresano@unizar.es

"Department of Clinical Biochemistry, "Miguel Servet" Universitary Hospital, Paseo Isabel La Católica 1-3, 50009, Zaragoza, Spain

${ }^{d}$ LCABIE, IPREM UMR 5254, CNRS - Université de Pau et des Pays de l'Adour, 64053, Pau cedex 9, France since $2010 .^{2}$ At present, a large number of biomarkers are detected in DBS, not only for screening for metabolic diseases in newborns, ${ }^{3}$ but also for other purposes such as therapeutic drug monitoring, ${ }^{4}$ pharmacokinetics, ${ }^{5}$ or toxicological ${ }^{6,7}$ and forensic studies. ${ }^{6}$

The reason for this wide acceptance of DBS sampling is certainly related to its inherent advantages in different aspects, which have recently awaken the interest of the pharmaceutical sector in this methodology, thus contributing to its growing success. $^{8,9}$ In this regard, minimally invasive collection of capillary blood via a finger or heel stick is probably the most important advantage, especially when applied to children under 2 years of age, for whom collection of venous blood is much more traumatic. Additionally, the simplicity of the process for preparing DBS specimens allows for non-specialized personal to carry out this procedure, even in unsupervised contexts such as at the patients' homes. The low amount of sample required to prepare a DBS is also advantageous in other situations such as discovery stage pharmacokinetics, where the use of DBS enables the pharmaceutical companies to better comply with the 3R's 
(Reduce, Refine, Replace) doctrine with respect to animal experiments (particularly when small rodents are deployed), allowing for serial sampling from a single individual. ${ }^{\mathbf{1 0}}$

As a second main advantage, the ease of transport and conservation of DBS is also remarkable. Due to the diversity of analytes that can be targeted in blood samples, no general conclusions can be drawn about the stability of the DBS specimens with time and/or storage conditions, as those might depend on the particular analyte considered. However, several studies indicate that the filter paper has a stabilizing effect on the sample, ${ }^{11}$ such that DBS specimens do not normally require very stringent measures for conservation and/or transport such as liquid samples do (i.e. deep freezing at $-20{ }^{\circ} \mathrm{C}$ to $-80{ }^{\circ} \mathrm{C}$ ). In fact, conservation at room temperature and for large periods of time is possible for many analytes including trace elements, ${ }^{12,13}$ so that DBS delivery can be carried out even by regular mail.

These features have obvious economical and practical implications. For instance, the ease of sample collection and the low cost of preservation and delivery can be particularly important for bringing mass screening studies to developing countries or remote locations, or for improving results of epidemiological studies for which the number of volunteers is low, as participants could donate samples without the need to go to the clinic. ${ }^{\mathbf{1 2}}$ Moreover, the stability of DBS and the low storage requirements make the use of these specimens much better suited for biobanking ${ }^{\mathbf{1 4}}$ than liquid samples. In conclusion, it seems clear from all of the above that extending the use of DBS sampling and analysis to as many analytes as possible would be of great interest from an economical, ethical, logistical and scientific point of view.

However, and in spite of these advantages, detractors of DBS sampling often cite the relative complexity added to the analytical process, as compared to collection and analysis of venous blood, as a major disadvantage for extending the use of this methodology. ${ }^{\mathbf{1 5}}$ In this regard two issues potentially problematic are normally highlighted: (i) difficulties for sample deposition and DBS formation, and (ii) the fact that a liquid sample is converted into a solid one, a priori, more difficult to analyze.

Although potentially problematic, sample deposition for DBS preparation is well established in the clinical practice as described in detail in specialized documents from the UK National Health System ${ }^{\mathbf{1 6}}$ or the US Clinical and Laboratory Standards Institute. ${ }^{17}$ When quantitative results are needed, the use of US Food and Drug Administration (FDA) approved papers ${ }^{18}$ with a constant sample retention per surface unit, is normally relied upon, although deposition of controlled volumes by using calibrated capillary pipettes is also accepted. ${ }^{9}$ More problematic is the influence that variable hematocrit contents, which directly influence sample viscosity, have on blood diffusion and formation of the DBS. Hematocrit is the portion of blood volume occupied by red cells and varies considerably for different individuals. ${ }^{19}$ The situation becomes especially problematic when commercial blood reference materials are compared with real samples. In this regard, Cizdziel showed, by using Scanning Electron Microscopy, that red cells were absent in DBS prepared from such a reference sample. ${ }^{13}$ As a result, diffusion properties of this material were totally different from those of real samples, which hampered its use for validation or calibration purposes when $\mathrm{Pb}$ determination in DBS by means of laser ablation-inductively coupled plasma-mass spectrometry (LA-ICP-MS) was considered. The development of analytical methods overcoming this difference is therefore highly desirable in the context of DBS quantitative analysis.

As for the second problematic issue, i.e. the fact that a DBS is actually a solid sample, this might indeed introduce additional problems if conventional analysis in solution is to be carried out. In these cases, an additional step for extracting the analyte out of the DBS is normally needed, which reduces sample throughput and increases contamination risks. ${ }^{15}$ However, and particularly in some areas of application such as trace elemental analysis, direct solid sampling techniques exist that allow for accurate and precise results to be obtained with none or minimal additional sample preparation. This is the case of LA-ICP-MS, a technique providing multielemental trace and ultra-trace as well as isotopic information, the use of which in all kinds of applications, ${ }^{20-22}$ including bioanalysis, ${ }^{23-28}$ has increased dramatically in the last few years. However, and in spite of its potential interest, there are only a few papers in the literature attempting the use of LA-ICP-MS for direct (multi) elemental analysis of DBS, ${ }^{\mathbf{1 3 , 2 9 , 3 0}}$ and none of them has been fully successful due to different reasons, as will be discussed in detail in Section 3.1.1.

It is the purpose of this paper to develop a methodology for the direct multielemental analysis of DBS at trace and ultratrace levels by means of LA-ICP-MS, trying to overcome the problem of differential responses for real samples and reference materials, and following as much as possible the recommendations for DBS collection common in the clinical practice as indicated, e.g., by the European Bioanalysis Forum. ${ }^{9}$ Four analytes covering a wide range of masses, $\mathrm{Cd}, \mathrm{Co}, \mathrm{Cu}$ and $\mathrm{Pb}$, were selected for proving the concept. Determination of these analytes is interesting in the clinical setting for different reasons, such as monitoring prosthesis degradation in implanted patients $(\mathrm{Co}),{ }^{31}$ revealing exposure to toxic metals $(\mathrm{Cd} \text { and } \mathrm{Pb})^{6}$ or because the elements targeted play a major role in a given medical condition ( $\mathrm{Cu}$ in the case of Wilson's Disease).${ }^{32}$ On the other hand, and considering recent advances in the field of isotopic analysis of biological samples that demonstrate the potential interest of such isotopic information for diagnosis of metal-related diseases, such as $\mathrm{Cu}$ isotopes and Wilson's disease, ${ }^{33,34}$ simultaneous determination of $\mathrm{Cu}$ isotope ratios with high precision by split-flow coupling of the LA system to a multicollector (MC)-ICP-MS was also attempted.

\section{Experimental}

\subsection{Chemicals and reagents}

All multielement and single element solutions used for preparation of matrix-matched calibration standards and preparation of the Pt internal standard solution were prepared by serial dilution of $1 \mathrm{~g} \mathrm{~L}^{-1}$ mono elemental standards for ICP-MS (Inorganic Ventures, Christiansburg, USA) with diluted nitric 
acid until a final concentration of $0.14 \mathrm{M} \mathrm{HNO}_{3}$. Pro-analysis nitric acid was purchased from Merck (Darmstadt, Germany). Ultrapure water with a resistivity of $\geq 18.2 \mathrm{~m} \Omega \mathrm{cm}$ was obtained from a Milli-Q system (Millipore, Île-de-France, France).

Whatman no. 903 paper cards (lot W-092) were obtained from Whatman International Ltd (Maidstone, U.K.). This is one of the two commercial paper sources registered by the FDA as Class II medical devices. ${ }^{18} 4.5 \mathrm{~cm}$ diameter discs fitting in the LA cell were manually cut out of totally empty cards (non-inked) using ceramic scissors.

\subsection{Samples and standards}

Four blood reference samples with certified values for the elements targeted in this study were analyzed for validation purposes. Clincheck Whole Blood Control level II was obtained from Recipe Chemicals + Instruments GmbH (Munich, Germany). Seronorm Trace Element Human Whole Blood, level II was obtained from Sero (Billingstad, Norway). Lyphochek Whole Blood Control, level 1 and level 2 were obtained from BioRad (Hercules, USA). All of these samples are provided as a lyophilized material and were reconstituted in purified water.

Real venous-blood samples from four healthy volunteers were obtained from the Hospital Universitario Miguel Servet (Zaragoza, Spain). Three blood samples were characterized for their trace elemental composition either via pneumatic nebulization (PN)-ICP-MS, after 20-fold sample dilution with $0.14 \mathrm{M}$ $\mathrm{HNO}_{3}$ (Cd and Co), or by means of high-resolution continuum source graphite furnace atomic absorption spectrometry (HR CS GFAAS), after 10-fold sample dilution with $0.14 \mathrm{M} \mathrm{HNO}_{3}(\mathrm{~Pb}$ and $\mathrm{Cu}$ ). Although precipitation of blood proteins was not evident to the bare eye after acid dilution of the samples, proper sample homogenization was ensured before analysis in order to avoid biased results caused by potential precipitates. For analysis by means of PN-ICP-MS, on the other hand, larger rinsing times between samples were allowed to prevent nebulizer clogging. In all cases external calibration with aqueous standards was used for analysis, with In added as an internal reference in the case of PN-ICP-MS.

Another real sample, which was also analyzed as described above, was used for the preparation of matrix-matched calibration standards. For this purpose, $1 \mathrm{~mL}$ aliquots were spiked with small amounts of multielement solutions prepared in 0.14 $\mathrm{M} \mathrm{HNO}_{3}$ so as to obtain a concentration range of spiked amounts ranging from 1 to $2000 \mu \mathrm{g} \mathrm{L} \mathrm{L}^{-1}$, depending on the element. The same dilution factor (lower than $2 \%$ ) was introduced in all cases. The standards prepared in this way were kept for $24 \mathrm{~h}$ in an automated orbital shaker before deposition on the clinical filter papers was carried out.

Filter paper discs for LA-ICP-MS analysis were prepared following two different procedures, depending on the way the internal standard used as a reference $(\mathrm{Pt})$ was added to the DBS specimens. In a first protocol, Pt was directly spiked into the liquid samples, standards and controls until a final Pt concentration of $100 \mu \mathrm{g} \mathrm{L}^{-1}$. For this purpose, Pt was added to the matrix-matched standards in a multielement solution together with the rest of the spiked elements as described above. The same protocol was used for spiking the samples and controls but with a monoelemental Pt solution. Afterwards, 5 or $15 \mu \mathrm{L}$ aliquots of the spiked standards, samples and controls were carefully deposited onto the surface of the filter paper discs, trying to fit as many blood spots as possible on each disc while ensuring enough blank space among them (see Fig. 1 for details). Samples were left to dry at room temperature for at least $4 \mathrm{~h}$ and, once dried, were kept in sealed plastic bags at room temperature until analysis.

In a second protocol, ${ }^{35}$ the filter paper discs used for sample deposition were impregnated with a $100 \mu \mathrm{g} \mathrm{L} \mathrm{L}^{-1}$ Pt solution and were left to dry at room temperature for at least $4 \mathrm{~h}$ before deposition of blood was carried out. Once dried, 5 or $15 \mu \mathrm{L}$ aliquots of matrix-matched standards, samples and controls (non-spiked with $\mathrm{Pt}$ ) were deposited onto their surface following the same procedure described above. Samples were left to dry at room temperature for at least $4 \mathrm{~h}$ and, once dried, were kept in sealed plastic bags at room temperature until analysis.

\subsection{Instrumentation and experimental setup for split-flow LA measurements}

All measurements were carried out using a Lambda 3 femtosecond laser ablation system (Nexeya SA, Canejan, France). This laser is fitted with a diode-pumped $\mathrm{Yb}: \mathrm{KGW}$ crystal laser source (HP2, Ampitudes Systèmes, Pessac, France) delivering 400 fs pulses. Three wavelengths can be selected: $1030 \mathrm{~nm}$ (fundamental), $515 \mathrm{~nm}$ ( $2^{\text {nd }}$ harmonic) and $257 \mathrm{~nm}$ (4 ${ }^{\text {th }}$ harmonic). The $1030 \mathrm{~nm}$ wavelength was used in this study. The laser source operates within a wide range of repetition rates (up to $100 \mathrm{kHz}$ ) and energy ( $<2 \mathrm{~mJ}$ per pulse below $1 \mathrm{kHz}$ and $<80 \mu \mathrm{J}$ per pulse at $100 \mathrm{kHz}$ at this wavelength), which represents a different approach in analytical applications where high energy and low repetition rate are commonly used. The laser beam is focused with a $100 \mathrm{~mm}$ objective, and it can be rapidly moved (up to $2 \mathrm{~m} \mathrm{~s}^{-1}$ ) with high repositioning precision thanks to a $2 \mathrm{D}$ galvanometric scanning module fitted to the optical line. Further details of a previous and similar model (operating in

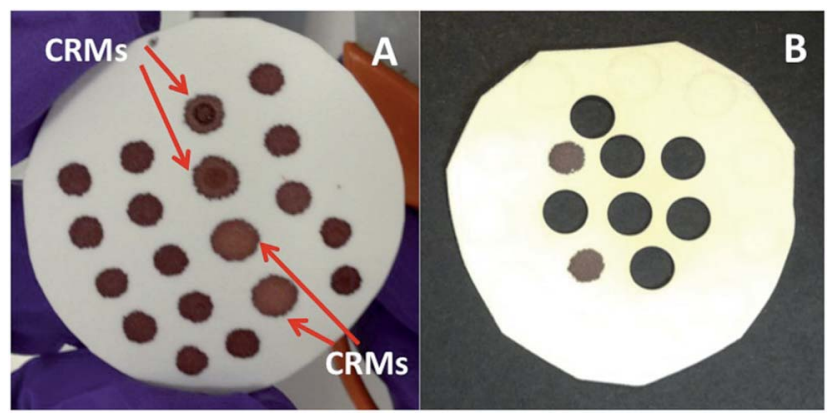

Fig. 1 Filter paper discs with $5 \mu \mathrm{L}$ dried blood spots (DBS) from different CRMs (highlighted in the figure) and real samples (spiked and unspiked). (A) Before ablation. The different dispersion properties of the CRMs and the real samples can be clearly appreciated; (B) after ablation. Each ablated area completely covers the DBS plus a variable part of blank paper depending on the DBS size. 
the IR region only) of this laser ablation system are described elsewhere. ${ }^{36,37}$

Splitting of the carrier gas flow through the LA system has been previously used for simultaneous isotopic and elemental analysis using two ICP-MS systems, ${ }^{38}$ and this possibility was also tested in the present work. $\mathrm{Cu}$ isotopic analysis was carried out on a Nu Plasma HR-MC-ICP-MS instrument ( $\mathrm{Nu}$ instruments, Wrexham, UK), while multielemental analysis was carried out on an Element XR sector field (SF) ICP-MS instrument (Thermo Scientific, Germany) fitted with a Jet Interface for improved sensitivity. This Jet Interface consists of a high capacity dry interface pump and a specially designed set of cones. Additionally, $\mathrm{N}_{2}$ has to be added to the dry LA aerosol

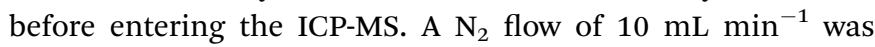
added in all the experiments carried out in this work. The LA coupling to the two ICP-MS systems was implemented using two $10 \mathrm{~m}$ long antistatic tubes (PTFE electroconductive, Fisher Bioblock, Illkirch Cedex, France) of $6 \mathrm{~mm}$ external diameter and $4 \mathrm{~mm}$ internal diameter into the ICP torches of both instruments, using He as the carrier gas. The He carrier gas flow was split after the LA sample cell with a Y-piece. To adjust sensitivity, $80 \%$ of the He flow was directed to the MC-ICP-MS while the remaining $20 \%$ was sent to the SF-ICP-MS. Considering the high amount of sample ablated and transported to the instruments per second, especially to the MC-ICP-MS, a glass fiber filter (37 $\mathrm{mm}$ diameter Millipore cassette partially filled with chromatographic grade glass wool) was placed before the entrance to the ICP torch of this instrument to prevent clogging of the injector tube. A schematic drawing of this experimental setup is shown in Fig. 2.

For isotope ratio measurements with the Nu instrument, a two-inlet torch was used to mix the laser-generated aerosol together with a liquid aerosol (nebulized by means of a pneumatic $200 \mu \mathrm{L} \mathrm{min}^{-1}$ micro-concentric nebulizer combined with a mini-cyclonic Cinnabar spray chamber) before introduction into the plasma. ${ }^{39,40}$ This dual-flow introduction system enables easy optimization of the MC-ICP-MS by nebulizing a suitable solution for tuning. Furthermore, during laser ablation analyses, the plasma was kept under wet conditions by the continuous nebulization of a Ni standard solution $\left(1 \mathrm{mg} \mathrm{L}^{-1}\right.$ in $0.14 \mathrm{M} \mathrm{HNO}_{3}$ ), which was used to correct for mass bias on $\mathrm{Cu}$.

Measuring conditions of the three instruments, summarized in Table 1, were adjusted for maximum sensitivity, stability and plasma robustness while minimizing the influence of interference on the analyte signals.

A high-resolution continuum source graphite furnace atomic absorption spectrometer ContrAA 700 from Analytik Jena (Jena, Germany) and a quadrupole-ICP-MS device (Nexion 300x, Perkin-Elmer, Waltham, USA) were used for validation purposes when required. A Sartorius (Goettingen, Germany) model BP211D analytical balance with a precision of $10^{-5} \mathrm{~g}$ was used for weighing.

\subsection{Procedure for simultaneous multielemental and $\mathrm{Cu}$ isotope ratio analysis of the samples of DBS by means of split flow LA-ICP-MS}

The instrumental parameters used for the analysis of the samples are summarized in Table 1. The filter paper discs containing the DBS specimens were mounted onto plastic holders with the form of an empty cylinder for introduction into the LA cell. For avoiding contamination issues, only the rim of the disc was in contact with the holder, so that complete ablation of the DBS specimens could be carried out without coablating the plastic. Each $5 \mu \mathrm{L}$ DBS was completely ablated by a series of 250-285 concentric circumferences (depending on its size), ablated from the inside to the outside of the blood spot

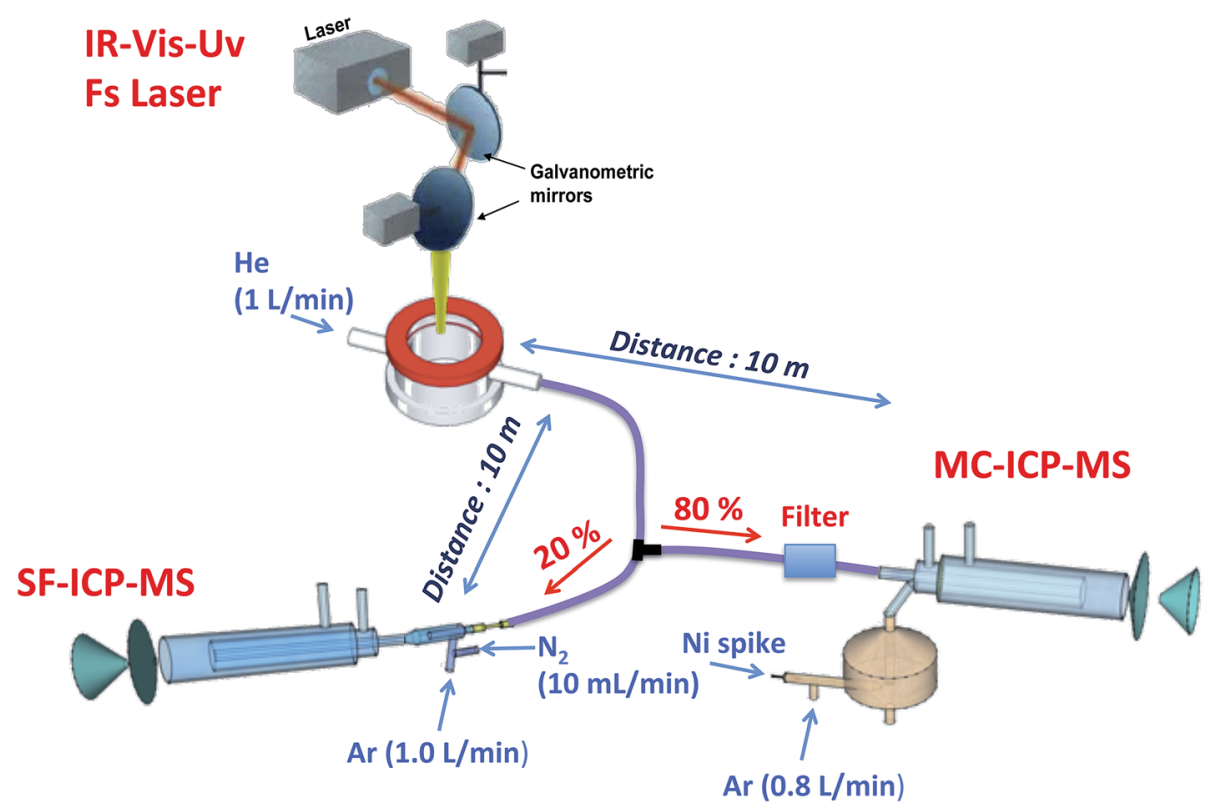

Fig. 2 Experimental setup for split-flow simultaneous elemental and isotopic analysis of the DBS samples by means of LA-ICP-MS, using two different types of ICP-MS devices (SF-ICP-MS and MC-ICP-MS). 
Table 1 Instrumental operating conditions used for split-flow simultaneous multielement and Cu isotopic analysis of the blood samples in the form of DBS

\begin{tabular}{ll} 
Laser ablation conditions & \\
\hline Laser ablation system & Lambda 3 fitted with a HP2 fs Laser source \\
Wavelength & $1030 \mathrm{~nm}$ \\
Pulse duration & $400 \mathrm{fs}$ \\
Repetition rate & $30000 \mathrm{~Hz}$ \\
Spot diameter (Airy $\left.1 / e^{2}\right)$ & $21 \mu \mathrm{m}$ \\
Energy/fluence & $135 \mu \mathrm{J} / 39 \mathrm{~J} \mathrm{~cm}$ \\
Scan speed $(y$-axis) & $10 \mathrm{~mm} \mathrm{~s}^{-1}$ \\
Stage speed $(x$-axis) & $30 \mu \mathrm{m} \mathrm{s}^{-1}$ \\
Ablation strategy & Circles $\left(5 \mathrm{or}^{-1} \mathrm{~mm} \varnothing\right)$, made with $250-285$ concentric circumferences \\
Transport gas & $\mathrm{He}, 1 \mathrm{~L} \mathrm{~min}^{-1}$
\end{tabular}

Element XR SF-ICP-MS

RF power

Ar plasma/auxiliary flow rates

Sampling cone and skimmer

$\mathrm{N}_{2}$ flow for jet interface

$\%$ of total He flow split

Lens voltages

Scanning mode

Settling time

Dwell time per acquisition point

Acquisition points per peak

Mass window scanned

Acquisition time per replicate

Resolution mode

Nuclides monitored

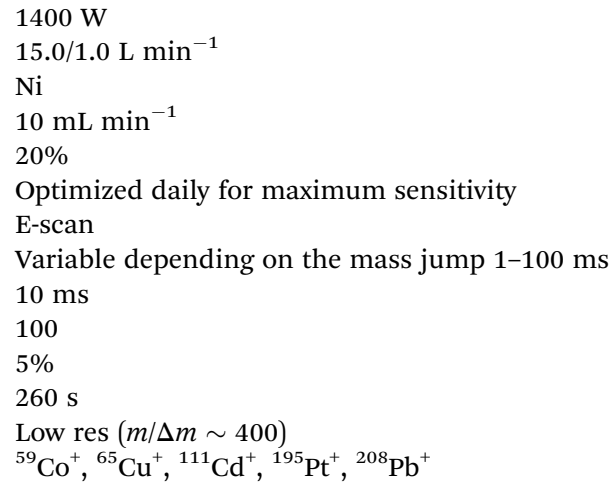

Nu MC-ICP-MS

RF power

Instrument resolution

Source slit width

Alpha 1 slit

Alpha 2 slit

Integration time

Plasma gas flow rate

Auxiliary gas flow rate

Nebulizer pressure

$\%$ of total He flow split
$1300 \mathrm{~W}$

Pseudo-high resolution $\sim 5700$ (edge resolved power) $0.05 \mathrm{~mm}$

$80 \mathrm{~mA}$

$90 \mathrm{~mA}$

$5 \mathrm{~s}$

13.0 $\mathrm{L} \mathrm{min}^{-1}$

$0.80 \mathrm{~L} \mathrm{~min}^{-1}$

$28.8 \mathrm{psi}$

$80 \%$

Faraday cup configuration

\begin{tabular}{lllllllllllllllll}
\hline $\begin{array}{l}\text { Collector } \\
m / z\end{array}$ & H7 & H6 & H5 & H4 & H3 & H2 & H1 & Ax & L1 & L2 & IC0 & L3 & IC1 & IC2 & L4 \\
& & & & & & 63 & & & 62 & & &
\end{tabular}

(see Fig. 3). For every replicate measurement a transient signal of 200-260 seconds duration was obtained. For quantitative analysis with the Element XR SF-ICP-MS instrument, integration of this transient signal was carried out for quantification purposes as described in detail in Section 3.1.2. For $\mathrm{Cu}$ isotopic analysis with the Nu MC-ICP-MS, on the other hand, a linear regression slope (LRS) method was used, where the signal intensities for ${ }^{65} \mathrm{Cu}$ were plotted against the signal intensities for ${ }^{63} \mathrm{Cu}$. The slope of the regression curve provides the raw ${ }^{65} \mathrm{Cu} /{ }^{63} \mathrm{Cu}$ ratio, which is later corrected for mass bias applying Russell's exponential law $^{\mathbf{4 1}}$ and the signal obtained for a $\mathrm{Ni}$ solution that was simultaneously nebulized and admixed with the ablated aerosol for this purpose. More details of this procedure are provided elsewhere. ${ }^{\mathbf{4 2 , 4 3}}$

\section{Results and discussion}

\subsection{Quantitative multielemental analysis}

3.1.1. Sample deposition on the filter paper and conditions for ablation of the DBS specimens. As discussed in the introduction, one of the main challenges faced when performing quantitative analyses of biological fluids dried on filter paper, such as DBS, is the occurrence of chromatographic effects both transversally and in-depth, which might affect samples from 


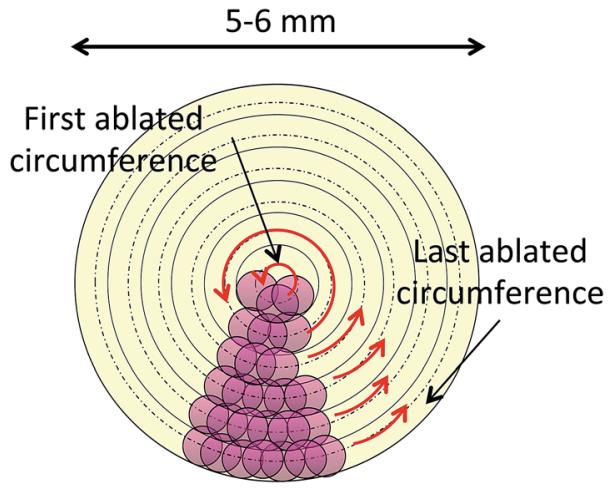

Fig. 3 Schematic representation of the ablation protocol used for analysis of the $5 \mu \mathrm{L}$ dried blood spots. $5-6 \mathrm{~mm}$ diameter craters (depending on the DBS size) were constructed by ablating 250-285 concentric circumferences from the inside to the outside of the DBS. All circles were ablated at the same speed.

different patients, standards and/or reference materials in a different way. ${ }^{13}$ The problem becomes especially significant when only a small part of the DBS is sampled, as is the case when conventional LA systems are used, because different results would be obtained depending on the sampling area selected. This problem is probably the reason why there are no studies published where quantitative elemental analysis of DBS specimens deposited on FDA approved filter paper cards has been successfully carried out by means of LA-ICP-MS.

To the best of the authors' knowledge, only three papers have been published attempting this challenge. The first of them explored the technique's possibilities and limitations, studying different DBS specimens prepared with real and reference samples. However, no attempt whatsoever to obtain quantitative information was carried out. ${ }^{13}$

On another paper by Hsieh et al. ${ }^{29} \mathrm{~Pb}$ could be quantified (in a reference sample) by means of conventional LA-ICP-MS in very small $(0.5 \mu \mathrm{L})$ dried blood spots deposited onto the surface of a hydrophobic (PTFE) filter membrane, and using aqueous standards for calibration. Using this sample deposition, complete desorption of the dried blood/standard droplets could be accomplished by programming a grid of $4 \times 5$ spots covering the surface of the droplet and using special ablation conditions: $2 \mathrm{~mm}$ laser defocus distance, maximum laser energy available, $20 \mathrm{~Hz}$ repetition rate and $8 \mathrm{~s}$ dwell time on each ablation spot. On a second paper by the same authors ${ }^{30}$ the same methodology was used to successfully quantify 8 additional elements in reference samples. For Cd determination, however, significant matrix effects were observed and matrix-matched calibration (obtained by serial dilution of a reference material) had to be deployed.

Although the method proposed by Hsieh et al. permitted to overcome potential chromatographic effects on the DBS by complete desorption of the sample and yielded good analytical results for the quantification of most of the target analytes, blood collection on PTFE slides instead of on FDA approved filter paper cards can be hardly considered as an ideal method for maintaining sample integrity over time and/or through delivery by regular mail, as the sample is not embedded in the PTFE membrane but only sticks to its surface. Moreover, accurate deposition of such small $(0.5 \mu \mathrm{L})$ sample volumes (needed for ensuring sample stability as well as minimal matrix effects and for maintaining short drying times) onto such a membrane does not seem straightforward and would probably imply collection of a small quantity of venous blood and sample deposition with a micropipette, which eliminates one of the main advantages of the DBS methodology. In any case, and even though the method by Hsieh might not be the most suited for routine analysis due to the potential difficulties discussed above, the idea of ablating the whole DBS for overcoming chromatographic effects and accurately quantifying the analytes irrespective of the sample origin (reference material or real blood samples) is worth exploring with specimens deposited on FDA-approved filter papers and following as much as possible the usual recommendations for bioanalysis in the clinical practice. ${ }^{9}$ While this possibility seems overly difficult with conventional LA systems delivering high energy laser pulses but at very low repetition rates (up to $20-50 \mathrm{~Hz}$ ), the use of the Lambda 3 laser, delivering lower energy laser pulses at very high repetition rates (up to $100 \mathrm{KHz}$ ), would potentially allow for this ablation strategy. ${ }^{34}$

For carrying out this test, deposition of relatively small DBS specimens was considered advantageous for keeping the ablation time at a manageable level, taking into account that the full paper depth should be pierced for avoiding any in-depth variations to affect the final quantification. As a result, deposition of $5 \mu \mathrm{L}$ blood spots, eventually allowing complete ablation of the DBS in a reasonable time (about $200 \mathrm{~s}$ as described below), was considered best for initial optimization of the analytical method.

As could be anticipated from the results published in the literature ${ }^{13}$ transversal chromatographic effects were visually detectable for the different types of samples under study. As shown in Fig. 1, DBS specimens of similar volume from reconstituted reference blood samples expand over a considerably larger area than those from real blood samples, with some of them even presenting two clearly differentiated areas with different colors. In that situation, complete ablation of the DBS specimen seems totally justified for obtaining comparable quantitative results for both the samples and the reference materials, one of the main goals of the work. For total ablation of the DBS, a series of contiguous concentric circles ablated from the inside to the outside of the blood spot was programmed (see Fig. 3), always ablating a small part of the blank paper surrounding the DBS for ensuring complete introduction of the blood sample into the ICP. Depending on the DBS size, different for CRMs and real samples as seen from Fig. 1, 250285 concentric circles were needed for complete sample ablation, thus providing transient signals of 200-260 seconds duration for the $5 \mu \mathrm{L}$ DBS specimens. In all cases, the complete sample was ablated with the same sampling speed.

3.1.2. Data treatment and influence of blood volume for DBS preparation. As could be expected from the different consistency and dispersion properties observed for the different samples and reference materials (Fig. 1), analysis of the 
transient signals obtained also revealed different analyte distributions along the surface of the DBS for the different specimens, which could be also different among the different target elements considered. This effect is illustrated for $\mathrm{Pb}$ in Fig. 4. For constructing this figure, the whole transient ${ }^{208} \mathrm{~Pb}^{+}$ signals acquired for $5 \mu \mathrm{L}$ DBS obtained from the reference and real samples were divided into slots of 10 seconds duration. Next, the ${ }^{208} \mathrm{~Pb}^{+}$signal intensity was integrated for these intervals and the results were normalized according to the $\mathrm{Pb}$ concentration in the samples, the values of which were available either from the certificate of analysis or from validation analysis carried out by means of GFAAS, as described in Section 2.2. Taking into account the speed of the laser translation movement, each $10 \mathrm{~s}$ interval was then assigned to its corresponding ablated corona. Results were depicted in the form of a bar graph, each bar representing the normalized area for an ablated corona, whose inner and outer radiuses are displayed in the $x$-axis. This graph thus represents an approximated radial

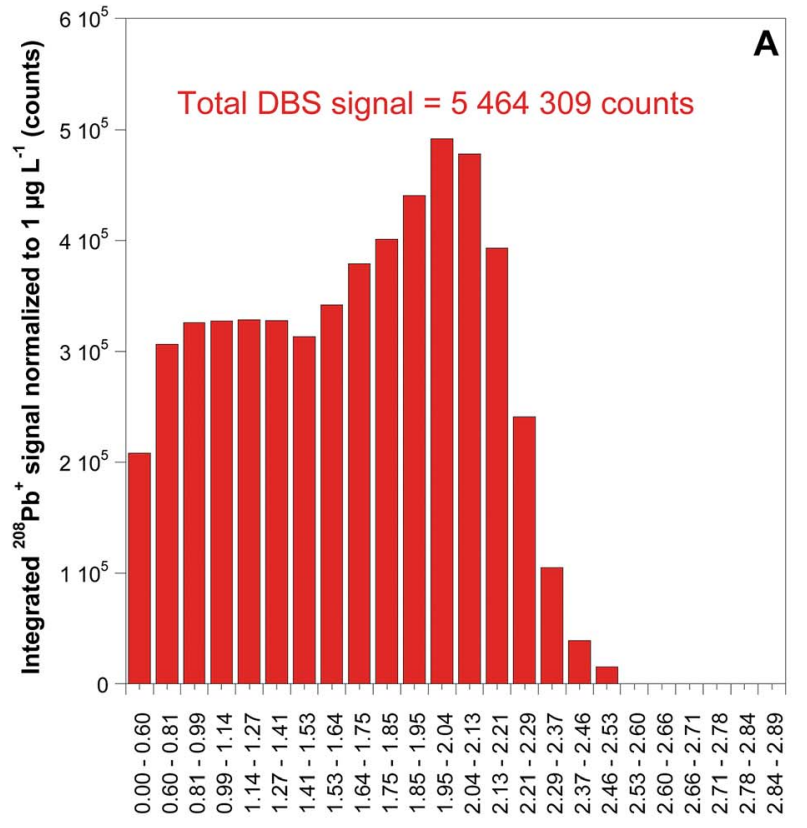

Radius interval for the ablated corona $(\mathrm{mm})$

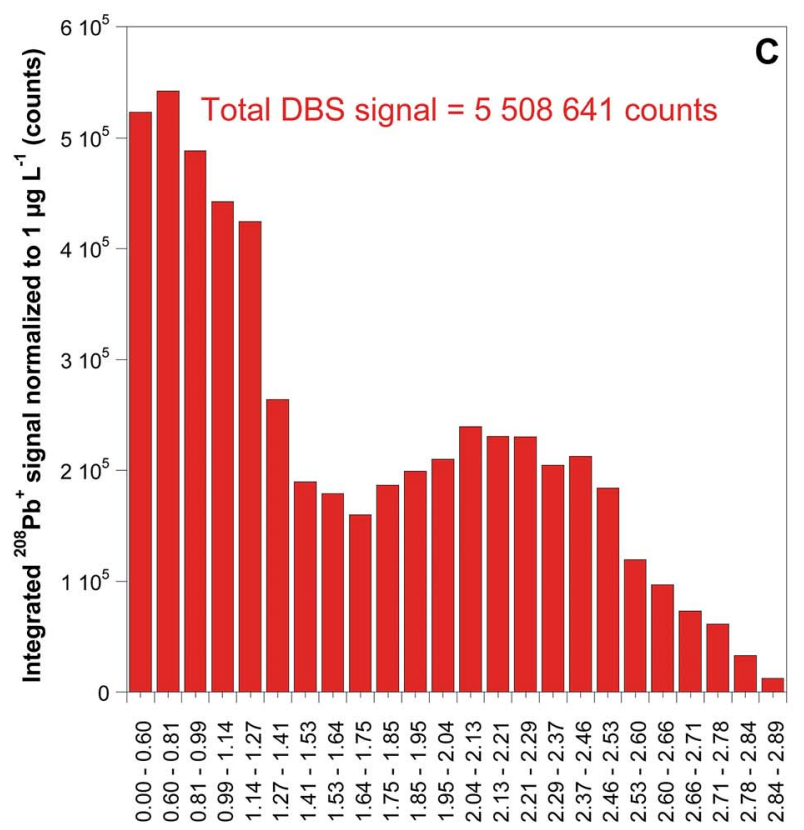

Radius interval for the ablated corona $(\mathrm{mm})$

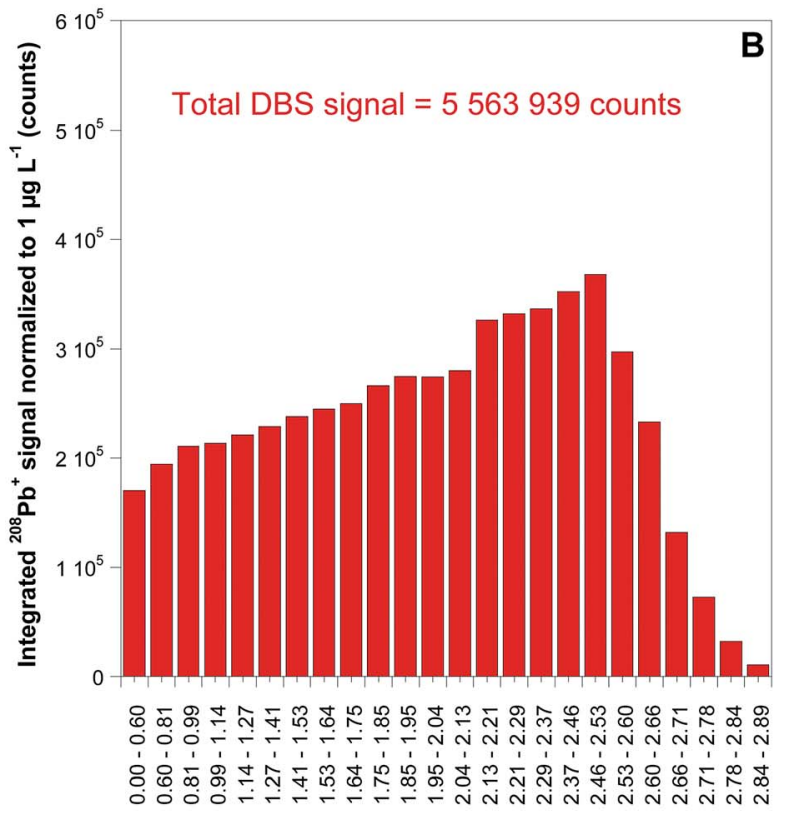

Radius interval for the ablated corona $(\mathrm{mm})$

Fig. 4 Approximated radial distribution of $\mathrm{Pb}$ in $5 \mu \mathrm{L}$ DBS obtained from: (A) a real sample (B) Lyphochek level I reference sample (C) Seronorm level II reference sample. Each bar represents the integrated ${ }^{208} \mathrm{~Pb}^{+}$signal, normalized for a concentration of $1 \mu \mathrm{g} \mathrm{L}{ }^{-1}$, for the ablated corona with internal and external radius indicated in the $x$-axis in the figure. 
distribution of $\mathrm{Pb}$ in the different DBS analyzed, normalized to a $\mathrm{Pb}$ concentration of $1 \mu \mathrm{g} \mathrm{\textrm {L } ^ { - 1 }}$ for facilitating comparison among the different specimens. The situation depicted in Fig. 4A and B represents the typical behavior observed for real samples and CRMs, respectively. The distribution pattern for $\mathrm{Pb}$ is similar in both cases, with $\mathrm{Pb}$ accumulation at the rim of the DBS. The analyte, however, migrates with the blood droplet, so that individual areas for each corona are lower on average for the reference materials but expand over a larger paper surface. In some extreme cases such as that depicted in Fig. 4C (that corresponds to one of the reference samples for which two clearly distinct areas could be visually detected in Fig. 1), $\mathrm{Pb}$ distribution was totally different with accumulation at the center of the DBS. In spite of these facts, it is clear from the final results shown in these figures that, although the analyte distribution along the DBS might vary and the consistency might be totally different for samples and reference materials, the total normalized integrated signal for the different DBS specimens remains practically constant. Similar graphs were constructed for the rest of the analytes under study and similar conclusions were reached for all of them. As a result, and in principle, quantification should be possible for DBS analyses provided that total signal integration is performed.

At this point, the possibility for using blood volumes greater than $5 \mu \mathrm{L}$ was considered. In fact, although venous-blood samples were used in this work for optimization of the analytical method, sample volumes that could be easily picked in a finger or heel prick by using calibrated capillary pipettes should be deployed for the method to fully exploit the advantages of DBS sampling, thus having the chance of being implemented in the clinical practice. In this regard, it is interesting to highlight that there are commercially available calibrated pipettes accurately picking and delivering small sample volumes starting from $5 \mu \mathrm{L} \pm 0.3 \%,{ }^{44}$ thus making the proposed methodology suited for practical implementation with capillary blood collection. However, and considering the general practice in the clinical setting for neonatal screening studies, where larger volumes of capillary blood (up to 50-75 $\mu \mathrm{L}$ ) are normally collected, the possibility for using the same working strategy for larger DBS specimens was considered to be an interesting point for research.

For testing this possibility, $15 \mu \mathrm{L}$ DBS specimens were prepared from one real sample, following the same protocol deployed for $5 \mu \mathrm{L}$ DBS and detailed in Section 2.2. The total ablation of this $15 \mu \mathrm{L}$ DBS needed 365 concentric circles, providing transient signals of 430 seconds duration. Integration of the signals thus obtained was carried out in a similar way to that described before, and the results were compared to those obtained with $5 \mu \mathrm{L}$ DBS of the same sample for all the analytes. Comparison of these results is summarized in Table 2. As seen from this table, the average signal values obtained for $15 \mu \mathrm{L}$ DBS specimens correspond to roughly three times those observed for $5 \mu \mathrm{L}$ DBS, with deviations in the range of $10-15 \%$ that were improved down to less than $10 \%$ if internal standard standardization is carried out, as will be described in detail in Section 3.1.3. This proves that good results can be obtained with
Table 2 Average ratio $(n=3)$ between signals obtained for $5 \mu \mathrm{L}$ and 15 $\mu \mathrm{L}$ DBS specimens of a real blood sample by means of LA-SF-ICP-MS and \% deviation from the theoretical value of three, both without and with the use of Pt as an internal standard. The internal standard was added to the filter paper prior to the deposition of the sample

\begin{tabular}{llrrrr}
\hline & Internal standard & $\mathrm{Cd}$ & $\mathrm{Co}$ & $\mathrm{Cu}$ & $\mathrm{Pb}$ \\
\hline Ratio signal $5 \mu \mathrm{L} / 15 \mu \mathrm{L}$ & \multirow{2}{*}{ No IS } & 3.4 & 3.4 & 2.6 & 2.5 \\
\% Deviation & & 12.3 & 14.7 & -14.0 & -15.4 \\
Ratio signal $5 \mu \mathrm{L} / 15 \mu \mathrm{L}$ & \multirow{2}{*}{ Pt as IS } & 2.9 & 3.2 & 2.8 & 2.8 \\
\% Deviation & & -2.1 & 6.3 & -8.3 & -7.4 \\
& & & & & \\
\hline
\end{tabular}

the working methodology proposed irrespective of the blood volume deployed for depositing the DBS.

3.1.3. Calibration strategy. Taking into account our previous experience with dried urine spot (DUS) analysis, ${ }^{26}$ calibration with matrix-matched standards was considered in the first place, as heavy matrix effects were expected for this kind of sample if aqueous standards were deployed, particularly considering the large amount of sample ablated per second (about $25 \mathrm{ng} \mathrm{s}^{-1}$ of blood and $18 \mu \mathrm{g} \mathrm{s}^{-1}$ of filter paper). This possibility is not difficult to implement in this particular case as it is relatively easy for a clinical lab to obtain pooled venous blood samples from healthy patients with low levels of the target analytes and spike them with appropriate concentrations of these elements, or else to acquire commercially available reference blood standards (a feasible possibility as the method developed ensures the same response for real and reference samples). Preparation of these matrix-matched calibration standards is described in Section 2.2.

At this point, addition and use of an adequate internal standard (IS) was evaluated. Use of an IS is highly recommended when working with LA-ICP-MS for quantitative purposes for correcting for different ablation efficiencies or sensitivity drifts. ${ }^{24}$ For this purpose, an element present in all samples and standards for which concentration is constant or known in advance needs to be used. In this regard, and considering the good results obtained in our previous work with DUS, ${ }^{26}$ addition of a constant Pt amount to all samples and calibration standards was contemplated. This element was considered ideal also in this case, as it is not typically found in blood samples, and should not be affected by spectral overlaps when monitored in a blood matrix by means of ICP-MS. The challenge at this point is to find a way for spiking samples and standards with the IS but without compromising the inherent advantages of DBS sample collection.

Three different approaches for IS application on DBS have been described in the literature for analysis of biomolecules, as detailed in the work by Abu Rabie and co-workers: ${ }^{45}$ (i) direct application of the IS onto the DBS; (ii) addition of the IS to the blood matrix before spotting it onto the paper; and (iii) use of paper discs that were previously pretreated with IS. These options were thus evaluated in the context of the current work. Option (i) was not considered feasible with the means available at our lab. In the work by Abu Rabie, a very fine IS aerosol was obtained by using a piezoelectric system (Touch Spray) 
especially designed for the purpose, providing satisfactory results for quantitative bioanalysis after sample extraction. Unfortunately, the Touch Spray device is not available at our lab, and the application of the IS over the filter paper card already impregnated with different blood samples and standards with a simple pipette, or by a similar method, could lead to DBS blurring and sample mixing. From the other two application methodologies, option (iii) is the only one that is fully compatible with DBS sample collection. Self evidently, option (ii) is not applicable to direct sampling from finger tip blood droplets using calibrated pipettes, as described in the introduction, but it may be argued that, a priori and from an analytical point of view, option (ii) would be preferred, as it ensures that the IS is fully incorporated into the sample matrix together with the analyte before deposition on the filter paper. Hence, the performance of both options, (ii) and (iii), was compared in this work. The addition of the IS to the samples, calibration standards and reference materials following these two deposition methodologies is fully described in Section 2.2.

The way in which IS normalization was carried out was different depending on which method had been followed for IS deposition. For DBS where the IS was added to the liquid sample before deposition on the filter paper, the ${ }^{195} \mathrm{Pt}^{+}$signal was integrated in the same way as those recorded for the rest of the analytes. The response value for samples and standards was then obtained by dividing the analytes' integrated signals by the ${ }^{195} \mathrm{Pt}^{+}$integrated signal. This methodology, however, cannot be deployed when papers pretreated with the IS are used for DBS deposition. In this case, Pt is distributed along the whole filter paper surface and hence, the integrated ${ }^{195} \mathrm{Pt}^{+}$signal varies with the size of the DBS ablated, so that direct normalization of the analyte signals with the integrated ${ }^{195} \mathrm{Pt}^{+}$signal will lead to erroneous results. In order to avoid this potential problem, the average ${ }^{195} \mathrm{Pt}^{+}$signal recorded during the integration interval considered for the analytes was used for normalization instead.

The performance of the two different IS deposition methods considered was next compared following the protocol described above for data treatment. For this purpose, analysis of the Lyphochek Whole Blood Control level 2 reference material was carried out using the two different IS deposition strategies. This material was selected as it exhibits comfortable concentration levels for all the analytes. As seen for $\mathrm{Pb}$ in Fig. 5, satisfactory calibration curves were obtained with both approaches for all the analytes, showing good linearities $\left(R^{2}>0.99\right)$ in the concentration ranges monitored (spiked concentrations: 1-100 $\mu \mathrm{g} \mathrm{\textrm {L } ^ { - 1 }}$ for Cd and Co; 1-500 $\mu \mathrm{g} \mathrm{L}^{-1}$ for $\mathrm{Pb} ; 1-2000 \mu \mathrm{g} \mathrm{L}^{-1}$ for $\mathrm{Cu}$ ). Please notice that the slopes of both calibration curves should not be directly compared as the amount of Pt that is incorporated into the filter paper using options (ii) and (iii) is not necessarily the same, although it should remain constant for every particular option.

The results obtained after analysis of the reference material, on the other hand, are gathered in Table 3. As seen from this table, similar results, within experimental error, were obtained for all the analytes using both IS deposition methodologies. Moreover, results are in good agreement with the reference values (obtained from the certificate of analysis, when available,

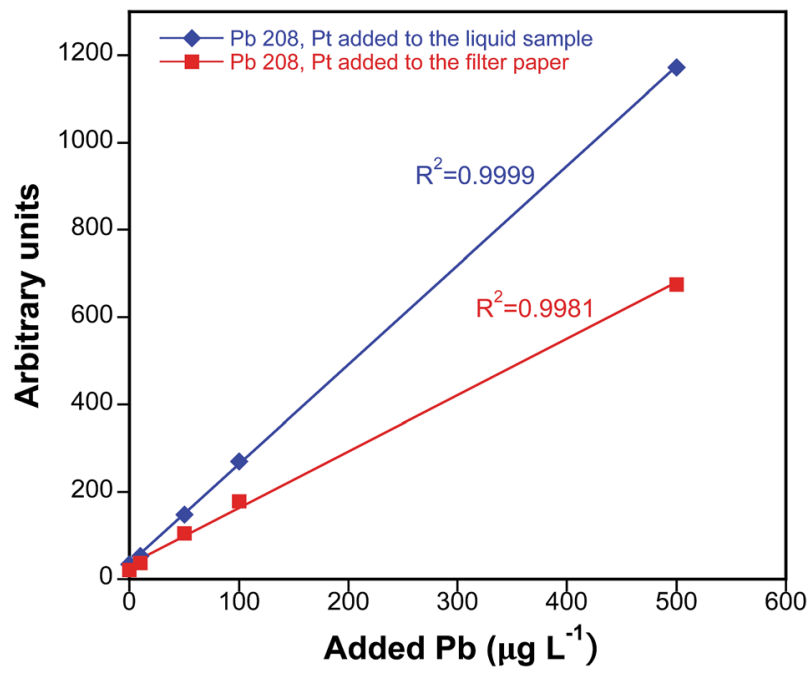

Fig. 5 Calibration curves for $\mathrm{Pb}$ obtained with matrix-matched standards (5 $\mu$ L DBS specimens) prepared from a real sample spiked with known $\mathrm{Pb}$ amounts, with either $\mathrm{Pt}$ added to the liquid sample (blue diamonds) or Pt previously added to the filter paper (red squares).

or from analysis of the blood samples by PN-ICP-MS or HR CS GFAAS, as described in Section 2.2), which demonstrates the validity of the analytical protocol developed. RSD values are typically below 10\%, except for Co (approx. 12\%), probably owing to the lower content of this element. Therefore, and considering that use of paper discs that were previously pretreated with the IS is the only methodology fully compatible with unsupervised sample collection schemes, such a method of IS addition was chosen for further experiments.

3.1.4. Analysis of real samples and reference materials. Results and figures of merit. Further validation of the LA-SFICP-MS method developed was carried out by analyzing a set of 3 real samples and 3 additional reference materials. The samples and those reference materials for which some reference values were not available from the certificate of analysis were also analyzed by means of either PN-ICP-MS (Co, Cd) or HR CS GFAAS $(\mathrm{Cu}, \mathrm{Pb})$, following the experimental procedures described in Section 2.2. Results for those analyses are gathered in Table 4. As can be seen, the results obtained by means of LA-SF-ICP-MS are satisfactory both in terms of accuracy (the

Table 3 Results of the analysis of Lyphochek Whole Blood Control level 2 with the two different methodologies tested for IS deposition. Uncertainty is expressed as $2 \mathrm{~s}(n=5)$. All values are expressed as $\mu \mathrm{g} \mathrm{L}^{-1}$

\begin{tabular}{llcl}
\hline Analyte & $\begin{array}{l}\text { Pt added to the } \\
\text { liquid sample }\end{array}$ & $\begin{array}{l}\text { Pt added to the } \\
\text { filter paper }\end{array}$ & $\begin{array}{l}\text { Reference } \\
\text { value }\end{array}$ \\
\hline $\mathrm{Cd}$ & $22.3 \pm 1.5$ & $21.6 \pm 1.4$ & $21.0 \pm 4.2^{a}$ \\
$\mathrm{Co}$ & $3.8 \pm 0.9$ & $4.2 \pm 0.8$ & $3.8 \pm 0.4^{b}$ \\
$\mathrm{Cu}$ & $584 \pm 81$ & $511 \pm 88$ & $526 \pm 24^{c}$ \\
$\mathrm{~Pb}$ & $286 \pm 22$ & $301 \pm 24$ & $297 \pm 59^{a}$
\end{tabular}

${ }^{a}$ Reference values available from the certificate of analysis. ${ }^{b}$ Reference value obtained by means of PN-ICP-MS. ${ }^{c}$ Reference value obtained by means of HR CS GFAAS. 
Table 4 Results and LOD for three reference materials (Seronorm, Clincheck level II and Lyphochek level I) and three real samples (M1, M2, M4) analyzed by means of LA-SF-ICP-MS following the experimental methodology described in Section 2.4. All values are expressed as $\mu \mathrm{g} \mathrm{L}^{-1}$. Uncertainty values are expressed as $2 \mathrm{~s}$

\begin{tabular}{|c|c|c|c|c|c|c|c|}
\hline & & Seronorm & Clincheck level II & Lyphochek level I & Real sample M1 & Real sample M2 & Real sample M4 \\
\hline \multirow[t]{2}{*}{ Cd } & Ref. value & $6.0 \pm 0.4^{a}$ & $3.14 \pm 0.63^{a}$ & $11.6 \pm 2.3^{a}$ & $0.15 \pm 0.02^{b}$ & $0.17 \pm 0.01^{b}$ & $0.21 \pm 0.03^{b}$ \\
\hline & $\begin{array}{l}\text { LA-SF-ICP-MS } \\
\text { LOD }\end{array}$ & \multicolumn{6}{|c|}{0.043} \\
\hline \multirow[t]{2}{*}{ Co } & $\begin{array}{l}\text { Ref. value } \\
\text { LA-SF-ICP-MS }\end{array}$ & $\begin{array}{l}6.1 \pm 0.4^{a} \\
6.8 \pm 0.8\end{array}$ & $\begin{array}{l}4.17 \pm 0.83^{a} \\
4.43 \pm 0.81\end{array}$ & $\begin{array}{l}2.2 \pm 0.8^{b} \\
2.0 \pm 0.7\end{array}$ & $\begin{array}{l}0.48 \pm 0.05^{b} \\
<\operatorname{LOQ}^{d}\end{array}$ & $\begin{array}{l}1.58 \pm 0.19^{b} \\
1.70 \pm 0.24\end{array}$ & $\begin{array}{l}0.76 \pm 0.07^{b} \\
<\mathrm{LOQ}^{d}\end{array}$ \\
\hline & LOD & \multicolumn{6}{|c|}{0.42} \\
\hline \multirow[t]{3}{*}{$\mathrm{Pb}$} & Ref. value & $393 \pm 21^{a}$ & $237 \pm 47^{a}$ & $117 \pm 23^{a}$ & $22 \pm 1.1^{c}$ & $28 \pm 2.1^{c}$ & $58 \pm 4.1^{c}$ \\
\hline & LA-SF-ICP-MS & $381 \pm 26$ & $259 \pm 26$ & $144 \pm 23$ & $24 \pm 2.7$ & $27 \pm 3.5$ & $55 \pm 5.3$ \\
\hline & LOD & \multicolumn{6}{|c|}{0.040} \\
\hline
\end{tabular}

${ }^{a}$ Reference values available from the certificate of analysis. ${ }^{b}$ Reference value obtained by means of PN-ICP-MS. ${ }^{c}$ Reference value obtained by means of HR CS GFAAS. ${ }^{d}$ Defined as ten times the standard deviation of the blank filter signals divided by the sensitivity.

small differences found when compared with the reference values are always within the uncertainty of the methods) and reproducibility. RSD values range between 3 and $9 \%$ in the majority of cases ( $77 \%$ of analyses), which seems fit-for-purpose in a clinical context.

As for limits of detection (LOD), these were estimated using the signals obtained for 5 calibration standards and also monitoring 5 blank filters (only Milli-Q water was deposited onto them). LODs were calculated as 3 times the standard deviation of the blank filter signals divided by sensitivity obtained for each of the calibration standards. The final value was obtained as the average of these 5 individual values and is also displayed in Table 4 for each of the target analytes. The higher values obtained for $\mathrm{Co}$ and $\mathrm{Cu}$ are probably related to the existence of spectral interference in the ICP-MS not resolved in the low resolution mode of analysis deployed $\left({ }^{38} \mathrm{Ar}^{23} \mathrm{Na}^{+}\right.$and ${ }^{43} \mathrm{Ca}^{16} \mathrm{O}^{+}$for ${ }^{59} \mathrm{Co}^{+}$and ${ }^{40} \mathrm{Ar}^{25} \mathrm{Mg}^{+}$for ${ }^{65} \mathrm{Cu}^{+}$, respectively). Although further experiments would be needed, it seems clear that some room for improvement exists in this regard if medium resolution is deployed for determination of these two analytes. In this work, however, it was preferred to work in the low resolution mode, thus ensuring a higher sensitivity, as the LODs achieved already suffice for the vast majority of the applications intended. Furthermore, and as shown in Table 4, very low detection limits were obtained for $\mathrm{Cd}$ and $\mathrm{Pb}$. This is not surprising considering the extremely high sensitivity that can be achieved with the experimental setup deployed, especially the LA unit that is able to ablate a very high amount of sample per second. It is worth indicating at this point that all results were obtained with the split-flow experimental setup described in Section 2.4, i.e., by only directing $20 \%$ of the LA ablated aerosol into the SF-ICP-MS instrument. Although one might consider that, by directing the full amount of ablated aerosol into the SF-ICP-MS instrument LODs could be somewhat improved, it is our belief that this would probably lead to increased matrix effects due to the high amount of blood and, especially, paper matrix introduced into the ICP-MS per second, as observed for $\mathrm{Cu}$ isotopic analysis in the MC-ICP-MS instrument (see Section 3.2 for more details). As a result, and even if some additional experiments would be needed to exactly determine how much sample matrix can be efficiently dealt with by the ICP-MS, we believe that the LODs provided can be considered as a realistic estimation of what can be achieved with this method of analysis. As previously indicated, only some improvements coming from the use of higher resolution modes for interfered elements could be expected.

\subsection{Simultaneous $\mathrm{Cu}$ isotopic analysis by means of split- flow LA-MC-ICP-MS}

As briefly discussed in the introduction, our recent experience in the field of isotopic analysis of biological samples has demonstrated the potential interest of such isotopic information, combined with quantitative information for the same elements, for diagnosis of metal-related diseases such as Wilson's disease and $\mathrm{Cu}$ isotopes. ${ }^{33,34}$ Based on these results, determination of $\mathrm{Cu}$ isotope ratios with high precision in the same DBS specimen used for quantitative analysis by split-flow coupling of the LA system to a MC-ICP-MS was attempted in this work, as a means to provide an analytical method capable of maximizing the information extracted from a single blood spot that might be, for instance, archived in biobanks.

Unfortunately, the challenges related to this task are noteworthy. To start with, previous results obtained for $\mathrm{Cu}$ isotopic determination in serum samples ${ }^{33}$ showed that, for discriminating patients with a certain medical condition related to $\mathrm{Cu}$ metabolism from healthy individuals, the method of analysis deployed must provide uncertainty budgets below about 0.5 delta units (2s), or different populations may overlap. For achieving this goal, serum samples were acid-digested in the cited work and $\mathrm{Cu}$ was separated from the sample matrix by ionexchange chromatography, which permitted to virtually eliminate $\mathrm{Na}$ from the measuring solutions. In fact, $\mathrm{Na}$ is found at high concentrations in serum and blood samples and causes significant interference problems in the ICP-MS that affect both precision and accuracy for $\mathrm{Cu}$ isotope ratio determination. In 
particular, $\mathrm{Na}$ is at the origin of the ${ }^{40} \mathrm{Ar}^{23} \mathrm{Na}^{+}$interference on the ${ }^{63} \mathrm{Cu}^{+}$signal. Considering that $\mathrm{Na}$ concentration in serum or blood of healthy patients is about 2000 times higher than that of $\mathrm{Cu}$ (even higher for Wilson's disease patients), the significance of this interference is very remarkable for this kind of analysis, where extremely good precision and accuracy values are needed.

When LA is used for sampling dried matrix spots for which minimal sample pre-treatment is aimed at, chemical analyte isolation from the sample matrix before analysis is not feasible and other ways to circumvent the problem of the ${ }^{40} \mathrm{Ar}^{23} \mathrm{Na}^{+}$ overlap must be found. For this purpose, a method frequently used in the past when instruments fitted with reaction or collision cells were not available yet was first tested in this work. Weakly bonded polyatomic interferents such as ${ }^{40} \mathrm{Ar}^{23} \mathrm{Na}^{+}$are believed to be formed by condensation reactions in the interface region of the ICP-MS, ${ }^{46}$ and the use of mixed-gas plasmas (obtained by addition of molecular and inert gases to the coolant, nebulizer or auxiliary Ar gas flows) has been found to reduce the extension in which these condensation reactions occur without affecting analyte sensitivity to a large extent. ${ }^{47}$ In this particular case, $\mathrm{N}_{2}(\mathrm{~g})$ was added in the torch injector of the ICP-MS, a solution that had proved successful in the past for reducing the ${ }^{40} \mathrm{Ar}^{23} \mathrm{Na}^{+}$interference on ${ }^{63} \mathrm{Cu}^{+}$for seawater analysis. ${ }^{48}$ Moreover, addition of $\mathrm{N}_{2}$ could also help to alleviate other matrix effects that might be present from the heavy sample matrix tackled in this work, through provision of more robust plasma conditions. ${ }^{49}$

This possibility was first tested with aqueous solutions, without any laser coupling. A two-inlet torch was used to introduce the Ar flow needed for nebulizing the aqueous solutions (first port), while $\mathrm{N}_{2}$ was added through the second port into the MC-ICP-MS torch, together with He to mimic the conditions used for LA analysis. In particular, three different solutions were prepared for exploring the effect of $\mathrm{N}_{2}$ addition on the measured Cu ratios (i) $200 \mu \mathrm{g} \mathrm{L} \mathrm{L}^{-1} \mathrm{Cu}$; (ii) $400 \mathrm{mg} \mathrm{L}^{-1} \mathrm{Na}$ and (iii) $200 \mu \mathrm{g} \mathrm{L}{ }^{-1} \mathrm{Cu}+400 \mathrm{mg} \mathrm{L}^{-1} \mathrm{Na}$, the latter solution showing similar concentration ratios for $\mathrm{Cu}$ and $\mathrm{Na}$ to those observed in real blood samples. These solutions were measured in the MC-ICP-MS at different $\mathrm{N}_{2}$ flow rates added to the main $\mathrm{He}$ and Ar flows, which were also finely optimized for each experiment together with the ion optics to avoid reflected power in the instrument to increase too much. The results of these experiments are summarized in Fig. 6.

As seen from Fig. 6A, addition of $\mathrm{N}_{2}$ to the ICP-MS results in a clear reduction of ${ }^{40} \mathrm{Ar}^{23} \mathrm{Na}^{+}$interference on ${ }^{63} \mathrm{Cu}^{+}$, as shown by the increasing ratio obtained for signals monitored at $m / z=63$ in solution (i), containing only $\mathrm{Cu}, v s$. solution (ii), containing only $\mathrm{Na}$, when increasing amounts of $\mathrm{N}_{2}$ were added to the system. Unfortunately and as also shown in Fig. 6A, this is accompanied by a significant reduction of analyte sensitivity, reaching a factor of 2 for a flow of $\mathrm{N}_{2}$ of $20 \mathrm{~mL} \mathrm{~min}^{-1}$. On the other hand, the effect of the $\mathrm{N}_{2}$ flow added on the uncorrected $65 / 63$ isotope ratios is displayed in Fig. 6B. As seen from this figure, 65/63 ratios obtained for solution (iii), containing both $\mathrm{Cu}$ and $\mathrm{Na}$, are biased low when no $\mathrm{N}_{2}$ is added to the system due to the formation of ${ }^{40} \mathrm{Ar}^{23} \mathrm{Na}^{+}$polyatomic species. Even though addition of $\mathrm{N}_{2}$ significantly improves the 65/63 ratio
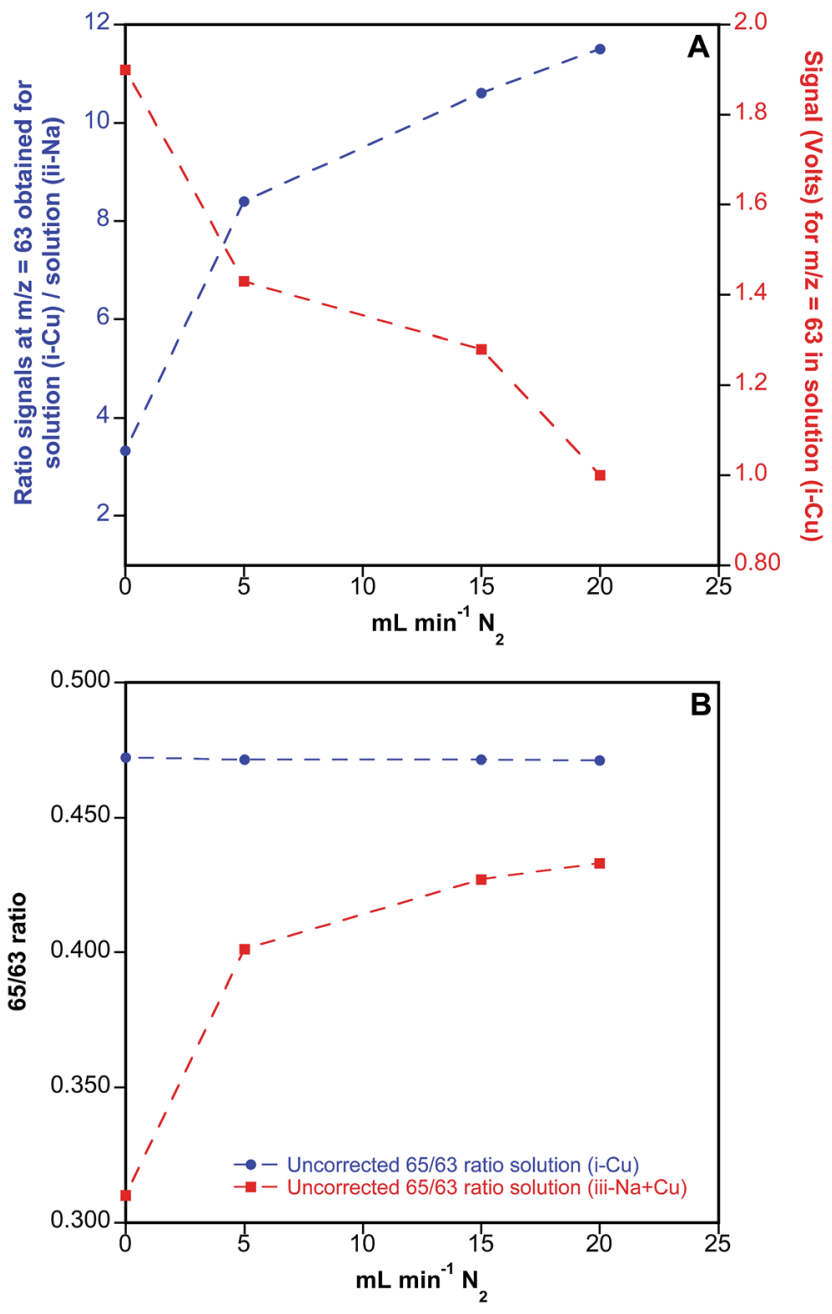

Fig. 6 Experiments for reduction of the ${ }^{40} \mathrm{Ar}^{23} \mathrm{Na}^{+}$interference on the ${ }^{63} \mathrm{Cu}^{+}$signal through addition of $\mathrm{N}_{2}$ to the plasma in the Nu MC-ICPMS instrument. (A) Left $y$-axis: ratio for the signals monitored at $m / z=$ 63 obtained for solution (i), containing $200 \mu \mathrm{g} \mathrm{L}^{-1} \mathrm{Cu}$, vs. solution (ii), containing $400 \mathrm{mg} \mathrm{L}^{-1} \mathrm{Na}$; right $y$-axis: signal intensity obtained at $\mathrm{m} / \mathrm{z}$ $=63$ for solution (i). (B) Uncorrected $65 / 63$ ratios obtained for solution (i) and for solution (iii), which contains $200 \mu \mathrm{g} \mathrm{L}^{-1} \mathrm{Cu}+400 \mathrm{mg} \mathrm{L}^{-1} \mathrm{Na}$.

obtained for this solution, the results never match those obtained for solution (i), containing only $\mathrm{Cu}$, indicating that the interference is only partially resolved by using this methodological approach. As a result, and considering also the reduction in sensitivity introduced by the addition of $\mathrm{N}_{2}$ to the system, this working methodology was disregarded for further experiments with DBS specimens.

Being the previous methodology unsuccessful for completely eliminating the influence of the ${ }^{40} \mathrm{Ar}^{23} \mathrm{Na}^{+}$interference on the ${ }^{63} \mathrm{Cu}^{+}$signal, the same approach that had provided good results for the analysis of DUS in ref. 34, i.e. working at pseudo high resolution mode and measuring the signals at the left side of the peak, was used for the rest of the experiments. Although this working methodology permitted to circumvent the interference problem for urine samples in the cited work, this effect was achieved at the cost of reducing the analyte sensitivity by a factor of 10 , which ultimately resulted in a considerable loss of 
precision, and this was the reason why this methodological approach was not considered from the beginning.

In addition, the first experiences with the DBS samples and the LA system showed that important matrix effects appear in the MC-ICP-MS instrument when these specimens were analyzed using the ablation conditions described in Section 2.3, selected for achieving adequate sensitivity and reasonable analysis time. In particular, the high amount of sample ablated per second and the fact that $80 \%$ of the ablated aerosol was directed to the MC-ICP-MS caused significant signal suppression in this instrument (directly observed in the ${ }^{62} \mathrm{Ni}^{+}$signal used for mass bias correction) and led to torch injector clogging after just two or three DBS ablations. To address the problem of clogging, a glass fiber filter (see Section 2.3. for details) had to be placed just before the entrance of the ablated aerosol to the instrument torch (see Fig. 2), which permitted to work without severe sensitivity fluctuations in sessions of more than $8 \mathrm{~h}$. On the other hand, and as for the signal suppression observed for ${ }^{62} \mathrm{Ni}^{+}$in the MC-ICP-MS, ablation of a blank filter permitted to conclude that most suppression effects came from the filter paper. This fact is advantageous as a similar response in the ICP-MS can be expected for all the DBS samples then, despite the possible matrix differences found in the original blood samples.

A specific study for determining the best precision achievable under laboratory conditions by means of this technique was carried out next. For this purpose, a set of 5 different DBS specimens from a healthy volunteer with a $\mathrm{Cu}$ content of about $1 \mathrm{mg} \mathrm{L}^{-1}$ was analyzed. The first issue taken into consideration was the way of treating the data for correcting 65/63 isotope ratios for mass bias, and two different approaches were also tested in this occasion: linear regression slope (LRS) and pointby-point methodology. ${ }^{34}$ In the LRS method, first proposed by Fietzke, ${ }^{42,43}$ the raw ${ }^{65} \mathrm{Cu} /{ }^{63} \mathrm{Cu}$ ratio is estimated as the linear regression slope of the graphical representation of ${ }^{65} \mathrm{Cu} v s .{ }^{63} \mathrm{Cu}$ signals, which is later corrected for mass bias applying Russell's exponential $\mathrm{law}^{41}$ and the signal obtained for the Ni solution simultaneously nebulized and admixed with the ablated aerosol. Conversely, in the point-by-point strategy the $65 / 63$ ratio for every individual measurement ( 1 value every 5 seconds) is calculated using the signal intensities (blank-corrected) for ${ }^{65} \mathrm{Cu}$ and ${ }^{63} \mathrm{Cu}$, and those ratios are averaged at the end for the complete transient signal. More details on these correction strategies can be found elsewhere. ${ }^{34,43,50}$ The results of this experiment showed that, as was the case for the analysis of DUS, ${ }^{34}$ the linear regression slope (LRS) method provides much better precision for the transient signals obtained than the traditional way to process data based on the use of a point-bypoint methodology. The results obtained with the LRS method are displayed in Table 5 . As can be seen, RSD values on the corrected ratios of about $800-1500 \mathrm{ppm}$ (internal precision) for ${ }^{63} \mathrm{Cu}^{+}$signal intensities of approx. $100-200 \mathrm{mV}$ corresponding to $\mathrm{Cu}$ contents of about $1 \mathrm{mg} \mathrm{L}^{-1}$ are obtained, while the pointby-point methodology provided RSD values up to 4 times worse for the same samples.

The delta values obtained for these specimens, using the Seronorm blood material as a reference, are also shown in
Table $5 \mathrm{Cu}$ isotopic composition of 5 different DBS specimens from a healthy volunteer with a $\mathrm{Cu}$ content of about $1 \mathrm{mg} \mathrm{L}^{-1}$, presented as $\delta^{65} \mathrm{Cu}$, the relative deviation (in per mil) versus a DBS specimen prepared with a reference sample (Seronorm). The RSD values indicate the internal precision of every measurement, and the overall RSD reflects the variation between the 5 specimens. The LRS method was used to correct for mass bias

\begin{tabular}{lcc}
\hline & $\delta^{65} \mathrm{Cu}(\%)$ & RSD $(\mathrm{ppm})$ \\
\hline DBS 1 & 1.57 & 1160 \\
DBS 2 & 1.33 & 1330 \\
DBS 3 & -2.17 & 1560 \\
DBS 4 & 0.21 & 800 \\
DBS 5 & -0.92 & 770 \\
Overall & 0.03 & 1570 \\
\hline
\end{tabular}

Table 5. As can be noticed, the variation found for every specimen is relatively large (from 1.6 to $-2.2 \%$ ), but the overall delta is very close to zero, as could be expected, because there is $a$ priori no reason to find differences in the $\mathrm{Cu}$ ratios for a healthy patient and a reference material that typically exhibits the "natural" Cu composition. This delta variation of almost $4 \%$ is obtained in the best analytical situation, if compared to the DBS from patients suffering from different medical conditions affecting $\mathrm{Cu}$ metabolism, for which much lower $\mathrm{Cu}$ contents can be expected. Nevertheless, these values are comparable to those obtained for analysis of DUS, ${ }^{34}$ indicating that the use of the split-flow methodology does not introduce additional sources of uncertainty to the method. While these values may still not be good enough to differentiate patients suffering from different diseases affecting $\mathrm{Cu}$ metabolism from healthy individuals, ${ }^{33}$ it seems clear that, in spite of the challenge that coupling the three instruments used in this work might represent, the method proposed for simultaneous isotopic and quantitative analysis of DBS samples is promising and can become a reality if the new MC-ICP-MS instrumentation available in the market providing better resolution values at a lower sensitivity cost is deployed for analysis. ${ }^{51}$

\section{Conclusions}

In this work it has been shown that simultaneous quantitative and isotopic information can be obtained after the direct analysis of DBS specimens by split-flow LA simultaneously coupled to a MC-ICP-MS and a SF-ICP-MS instrument, without degrading the analytical performance of each of the hyphenated techniques, which could be a very interesting alternative for maximizing the information that can be drawn from a single DBS specimen that might be, e.g., archived in a biobank or difficult to obtain again.

The selection of an ablation strategy implying the complete sampling of each DBS specimen has permitted to achieve for the first time similar analytical responses for DBS obtained from real and reference samples by means of LA-ICP-MS. In this way, calibration is simplified and can rely upon construction of regression curves based on in-house matrix-matched standards 
(prepared out of spiked pooled blood samples from healthy volunteers or from reference materials), leading to satisfactory results in terms of precision, accuracy and LOD for analysis of real blood samples in the form of DBS.

\section{Acknowledgements}

This work has been funded by the Spanish Ministry of Economy and Competitiveness (project CTQ2012-33494), the Aragón Government (Fondo Social Europeo), the Région Aquitaine and Feder.

\section{References}

1 R. Guthrie and A. Susi, Pediatrics, 1963, 32, 338-343.

2 P. A. Demirev, Anal. Chem., 2013, 85, 779-789.

3 J. J. Pitt, Clin. Biochem. Rev., 2010, 31, 57-68.

4 P. M. Edelbroek, J. van der Heijden and L. M. L. Stolk, Ther. Drug Monit., 2009, 31, 327-336.

5 P. Beaudette and K. P. Bateman, J. Chromatogr. B: Anal. Technol. Biomed. Life Sci., 2004, 809, 153-158.

6 C. P. Stove, A. S. M. E. Ingels, P. M. M. De Kesel and W. E. Lambert, Crit. Rev. Toxicol., 2012, 42, 230-243.

7 M. Resano, L. Rello, E. García-Ruiz and M. A. Belarra, J. Anal. At. Spectrom., 2007, 22, 1250-1259.

8 N. Spooner, Bioanalysis, 2010, 2, 1343-1344.

9 P. Timmerman, S. White, S. Globig, S. Lüdtke, L. Brunet and J. Smeraglia, Bioanalysis, 2011, 3, 1567-1575.

10 S. Tanna and G. Lawson, Anal. Methods, 2011, 3, 1709-1718. 11 T. W. McDade, S. Williams and J. J. Snodgrass, Demography, 2007, 44, 899-925.

12 J. C. Rockett, G. M. Buck, C. D. Lynch and S. D. Perreault, Environ. Health Perspect., 2004, 112, 94-104.

13 J. V. Cizdziel, Anal. Bioanal. Chem., 2007, 388, 603-611.

14 P. Elliott and T. C. Peakman, Int. J. Epidemiol., 2008, 37, 234244.

15 P. Abu-Rabie, Bioanalysis, 2011, 3, 1675-1678.

16 UK Newborn Screening Programme Centre, Guidelines for Newborn Blood Spot Sampling, UK National Screening Committee, February 2012, ISBN: 978-0-9562374-2-2, newbornbloodspot.screening.nhs.uk.

17 W. H. Hannon, V. R. De Jesús, M. S. Chavez, B. F. Davin, J. Getchell, M. Green, P. V. Hopkins, K. B. Kelm, B. Noorgaard-Pedersen, C. Padilla, E. Plokhovy, J. V. Mei and B. L. Therrell, Blood Collection on Filter Paper for Newborn Screening Programs; Approved Standard, Clinical and Laboratory Standards Institute (CLSI), Document NBS01-A6, West Valley Road, Suite 1400, Wayne, Pennsylvania 19087-1898 USA, 6 edn, 2013.

18 J. V. Mei, S. D. Zobel, E. M. Hall, V. R. de Jesús, B. W. Adam and W. H. Hannon, Bioanalysis, 2010, 2, 1397-1403.

19 A. J. Wilhelm, J. C. G. den Burger, R. M. Vos, A. Chahbouni and A. Sinjewel, J. Chromatogr. B: Anal. Technol. Biomed. Life Sci., 2009, 877, 1595-1598.

20 J. Pisonero, B. Fernández and D. Günther, J. Anal. At. Spectrom., 2009, 24, 1145-1160.

21 J. Koch and D. Günther, Appl. Spectrosc., 2011, 65, 155-162.
22 R. E. Russo, X. Mao, J. J. Gonzalez, V. Zorba and J. Yoo, Anal. Chem., 2013, 85, 6162-6177.

23 I. Konz, B. Fernández, M. L. Fernández, R. Pereiro and A. Sanz-Medel, Anal. Bioanal. Chem., 2012, 403, 2113-2125.

24 D. Hare, C. Austin and P. Doble, Analyst, 2012, 137, 15271537.

25 M. R. Flórez, M. Aramendía, M. Resano, A. C. Lapeña, L. Balcaen and F. Vanhaecke, J. Anal. At. Spectrom., 2013, 28, 1005-1015.

26 M. Aramendía, L. Rello, F. Vanhaecke and M. Resano, Anal. Chem., 2012, 84, 8682-8690.

27 W. Nischkauer, F. Vanhaecke, S. Bernacchi, C. Herwig and A. Limbeck, Spectrochim. Acta, Part B, 2014, 101, 123-129.

28 D. Pozebon, G. L. Scheffler, V. L. Dressler and M. A. G. Nunes, J. Anal. At. Spectrom., 2014, 29, 2204-2228.

29 H.-F. Hsieh, W.-S. Chang, Y.-K. Hsieh and C.-F. Wang, Talanta, 2009, 79, 183-188.

30 H.-F. Hsieh, W.-S. Chang, Y.-K. Hsieh and C.-F. Wang, Anal. Chim. Acta, 2011, 699, 6-10.

31 A. Sarmiento-González, J. M. Marchante-Gayón, J. M. Tejerina-Lobo, J. Paz-Jiménez and A. Sanz-Medel, Anal. Bioanal. Chem., 2008, 391, 2583-2589.

32 A. Ala, A. P. Walker, K. Ashkan, J. S. Dooley and M. L. Schilsky, Lancet, 2007, 369, 397-408.

33 M. Aramendía, L. Rello, M. Resano and F. Vanhaecke, J. Anal. At. Spectrom., 2013, 28, 675-681.

34 M. Resano, M. Aramendía, L. Rello, M. L. Calvo, S. Bérail and C. Pécheyran, J. Anal. At. Spectrom., 2013, 28, 98-106.

35 J. Mommers, Y. Mengerink, E. Ritzen, J. Weusten, J. van der Heijden and S. van der Wal, Anal. Chim. Acta, 2013, 774, 2632.

36 E. Ricard, C. Pécheyran, G. Sanabria Ortega, A. Prinzhofer and O. F. X. Donard, Anal. Bioanal. Chem., 2011, 399, 21532165.

37 C. Pécheyran, S. Cany, P. Chabassier, E. Mottay and O. F. X. Donard, J. Phys.: Conf. Ser., 2007, 59, 112-117.

38 H.-L. Yuan, S. Gao, M.-N. Dai, C.-L. Zong, D. Günther, G. H. Fontaine, X.-M. Liu and C. R. Diwu, Chem. Geol., 2008, 247, 100-118.

39 G. Ballihaut, L. Tastet, C. Pécheyran, B. Bouyssiere, O. Donard, R. Grimaud and R. Lobinski, J. Anal. At. Spectrom., 2005, 20, 493-499.

40 G. Ballihaut, F. Claverie, C. Pécheyran, S. Mounicou, R. Grimaud and R. Lobinski, Anal. Chem., 2007, 79, 68746880.

41 F. Vanhaecke, L. Balcaen and D. Malinovsky, J. Anal. At. Spectrom., 2009, 24, 863-886.

42 J. Fietzke, M. Frische, T. H. Hansteen and A. Eisenhauer, J. Anal. At. Spectrom., 2008, 23, 769-772.

43 J. Fietzke, V. Liebetrau, D. Günther, K. Gürs, K. Hametner, K. Zumholz, T. H. Hansteen and A. Eisenhauer, J. Anal. At. Spectrom., 2008, 23, 955-961.

44 http://www.hawksley.co.uk/blood_analysis/02i_pipettes/ index.shtml, last accessed November 2014.

45 P. Abu-Rabie, P. Denniff, N. Spooner, J. Brynjolffssen, P. Galluzzo and G. Sanders, Anal. Chem., 2011, 83, 87798786. 
46 H. Niu and R. S. Houk, Spectrochim. Acta, Part B, 1996, 51, 779-815.

47 B. S. Sheppard and J. A. Caruso, J. Anal. At. Spectrom., 1994, 9, 145-149.

48 M. J. Bloxham, P. J. Worsfold and S. J. Hill, Anal. Proc., 1994, 31, 95-97.
49 C. Agatemor and D. Beauchemin, Anal. Chim. Acta, 2011, 706, 66-83.

50 V. N. Epov, S. Berail, M. Jimenez-Moreno, V. Perrot, C. Pecheyran, D. Amouroux and O. F. X. Donard, Anal. Chem., 2010, 82, 5652-5662.

51 L. Yang, Mass Spectrom. Rev., 2009, 28, 990-1011. 